\title{
The application status and prospects of magneto-rheological fluid
}

\author{
Yuan Shu*, Ren Hongjuan, Luo Yiping, Xu Biao \\ Faculty of Automotive Engineering, Shanghai University of Engineering and Science, Shanghai, China \\ Email address: \\ yama123ys@126.com (Yuan Shu), 122587984@qq.com (Ren Hongjuan), lyp777@sina.com (Luo Yiping), \\ 846284570@qq.com (Xu Biao)
}

\section{To cite this article:}

Yuan Shu, Ren Hongjuan, Luo Yiping, Xu Biao. The Application Status and Prospects of Magneto-Rheological Fluid. International Journal of Materials Science and Applications. Vol. 3, No. 3, 2014, pp. 88-91. doi: 10.11648/j.ijmsa.20140303.13

\begin{abstract}
As a kind of new intelligent material, magneto-rheological fluid (MRF) has been receiving more and more attentions and applications. In this paper, the components of magneto-rheological fluid and its performance characteristics are mainly introduced. Besides, the authors analyze the advantages of MRF in practical application and its present situation of application in engineering. Finally, the application of MRF in engine dynamometer is studied in detail which will widen the application of magneto-rheological fluid.
\end{abstract}

Keywords: MRF, Performance Characteristics, Application, Engine Dynamometer

\section{Introduction}

Magneto-rheological fluid is consisted of magnetic particles, which is equipped with high permeability and low hysteresis characteristics, and non-magnetic base fluid. MRF mainly includes carrier fluid, paramagnetic or diamagnetic particles and additives ${ }^{[1]}$. When there is no magnetic field, MRF is a liquid that can free flow; While with the effect of magnetic field, it can change itself into so called 'similar solid' instantaneously. The viscosity of MRF increases rapidly so that its flow ability is lost and shows the behavior of Bingham fluid, and it also has an ability of anti-shear yield stress ${ }^{[2]}$.By adjusting the intensity of magnetic field, the viscosity and shear stress of MRF can been changed rapidly, sensitively and reversibly. As soon as magnetic field disappears, MRF will free flow rapidly, which is called MRF effect ${ }^{[3]}$.So in present, shock absorbers and power transmission device of luxury cars choose MRF to be used increasingly.with the fast response characteristics of magneto rheological fluid and magneto-rheological effect, in this paper, application of MRF in engine dynamometer is presented.

\section{Magneto-Rheological Fluid and Its Composition}

Magneto-rheological fluid is consisted of magnetic particles, carrier fluid and additives.

\subsection{Magnetic Particles}

Magnetic particles is the main parts of MRF, which can been made by ferromagnetic or paramagnetic particles. Currently, the mainly magnetic particles of MRF are ferrocobalt, iron nickel alloy, barium ferrite, strontium ferrite, carbonyl iron powder and so on. Compared with carbonyl iron powder, ferrocobalt and iron nickel alloy have a better rheological property, but because of its cheap price, carbonyl iron powder is widely used currently.

\subsection{Carrier Fluid}

The performance of carrier fluid has a direct influence on MRF, and it is the main component of magneto-rheological fluid. In the process of preparation of magneto-rheological fluid, it's common to use silicone oil or mineral oil as the carrier fluid. In addition, the options of carrier fluid also include high boiling synthetic oil, transformer oil, octane, toluene, and even pure water.

\subsection{Additives}

Additives is generally consisted of surface active agent, dispersant and stabilizer. Due to the high density of ferromagnetic particles and the low density of carrier liquid, the density difference between them leads to a settlement of 
magnetic particles of MRF, what is one of the main problems of the application of MRF. Therefore, one of the main roles of additives is to solve the problem of the settlement of MRF. Synthesizing current research status of this problem, we find the main solutions include:(1)Using composite magnetic particles. ferromagnetic particles is mixed with porous silica gel, and a process is done to make every particle has a certain thickness of binder on its surface and a solidification process is performed, composite magnetic particles are obtained after being reprocessed once more. Mixing this magnetic particles $(60 \mathrm{ml})$ with silicone oil $(96 \mathrm{ml})$ and siloxane $(8 \mathrm{ml})$ and then polishing for $10 \mathrm{~h}$, the MRF made by this way can have a better stability ${ }^{[4]}$. (2)Adding the surface active agent into magneto-rheological fluid. Surface active agent is an oligomer with two different part: lipophilic group and hydrophilic group, so it can change the surface properties of magnetic particles, increase particle volume and reduce the chance to attract or collide with each particle, so it is difficult to reunite them.

\section{The Performance Characteristics of MRF}

Generally, there are three operating modes of Magneto-rheological fluid: flow, shear and extrusion, and shear mode is used widely. Without using a magnetic field, MRF is the fluid whose particles inside distribute disorderly and freely, and it shows the characteristic of Newtonian fluid. While with the effect of magnetic field, the properties of MRF changes rapidly, and its performance characteristics changes instantaneously.

\subsection{The Rheology of MRF}

When there is no magnetic field, the smaller the viscosity of MRF is, the better the magneto-rheological effect is.

While with the effect of magnetic field, MRF changes itself from Newton fluid into Bingham fluid instantaneously. Because of the magnetic moment effect among magnetic particles, the rotation speed of particles is changed, so there is a friction between particles and carrier fluid, what may affect the viscosity of the magneto-rheological fluid, making its apparent viscosity increase significantly, which increases with the increase of the magnetic field. During this process, the viscosity of MRF changes continuously and steplessly, the entire conversion is very fast as well as under control, and energy consumption is also minimal.

\subsection{The Mechanical Properties of MRF}

The mechanical properties of MRF includes three stages: pre-yield, yield and post-yield. According to the constitutive properties of MRF, while with the effect of magnetic field, MRF shows a behavior of Bingham fluid, and there is a linear relationship between the shear stress and shear rate. So shear stress can be expressed as follows:

$$
\tau=\tau_{B}+\tau_{\eta}=\tau_{B}+\eta \gamma
$$

where, $\tau_{B}$ is regarded as the shear yield stress when a magnetic field effects on MRF, which relates to the magnetic field intensity $H$ and can be regulated; $\tau_{\eta}$ is the viscous damping force which is similar to a common fluid, it is a constant that has nothing to do with the magnetic field; $\eta$ is zero-field viscosity, which is independent on the magnetic field strength; $\gamma$ is shear strain rate.

Under the action of magnetic field, the shear stress of MRF increases significantly, and it increases with the increase of the magnetic field strength. Generally considered, there are some phenomenological models between yield shear strength and magnetic field strength, and exponential model considered as a good choice, and the index is about 1.7 1.8.

\subsection{The Magnetic Properties of MRF}

When external magnetic field strength is very small, for isotropic media, at any point of the magnetic medium, the magnetic field strength is proportional to the magnetization:

$$
\mathrm{M}_{\mathrm{s}}=\chi_{0} \mathrm{H}
$$

where, $M_{s}$ is magnetization; $\chi_{0}$ is magnetic susceptibility, which is a dimensionless number; $H$ is magnetic field strength.

With increasing of the magnetic field strength:

$$
\mathrm{M}_{\mathrm{s}}=\mathrm{B} / \mu_{0}-\mathrm{H}
$$

where, $B$ is magnetic induction intensity, $\mu_{0}$ is permeability of vacuum;

and:

$$
\begin{aligned}
& \mu=B / H \\
& \mu_{r}=\mu / \mu_{0}
\end{aligned}
$$

then:

$$
\mathrm{M}_{\mathrm{s}}=\left(\mu_{\mathrm{r}}-1\right) \mathrm{H}
$$

where, $\mu$ is permeability; $\mu_{r}$ is relative permeability. With the further increase of magnetic field intensity, MRF reaches full saturation.

\subsection{The Advantages of MRF}

As a kind of new intelligent materials, MRF has a transient response characteristic in millisecond. Compared with ERF, frist of all, MRF has a higher yield stress, generally greater about $80 \mathrm{MPa}$. secondly, the controllable temperature range of MRF is larger. Analyzing all kinds of magneto-rheological fluid temperature control ranges, we find that the total maximum temperature width can up to $-40^{\circ} \mathrm{C}-150^{\circ} \mathrm{C}$. In addition, compared with ERF, MRF also has the following advantages: a lower zero-field viscosity, a better stability, a faster response, a less dosage, and MRF 
also has some advantages of compact structure, light weight, high security. Moreover, MRF has a low requirement for the field which can activate and control it, the magnetic field made by basic electromagnetic induction loop can meet its need, so as long as there is an action of magnetic field, MRF will produce a stress field over dozens of KPs.

\section{The Engineering Application Status of MRF}

As a kind of new intelligent material, MRF is mainly applied to these constructions such as hydraulic device, clutch, brake, seal, buffing attachment, flexible fixture and so on, and its applications also involve electronics, aerospace, national defense, energy, chemical industry, environmental protection, metallurgy, machinery, instruments and meters, medical and health care, etc. Above all, the development and application of magneto-rheological fluid in transmissions and shock absorptions are particularly prominent.

\subsection{Magnetorheological Transmission}

Magnetorheological transmission is a new power transmission technology developed in the 1990s, its transmission theory is based on magneto rheological effect, magneto-rheological fluid is used as the transmission medium, by adjusting the strength of magnetic field to change the viscosity and yield stress of MRF in order to realize stepless change of rotation rate and torque.

Currently, the applications of MR transmission mainly focus on the power transmission device which is represented by brake and clutch. The Lord Company of USA has created the rotary brake, it's a device with follows advantages: controllable rotary resistance, stable operation, compact structure and low power consumption, so it has been used to bicycles and climb stairs style fitness equipment $^{[5]}$; Foister and Gopalswamy work in General Motors developed MRF and MRF clutch ${ }^{[6,7]}$; Yalcintas et al. ${ }^{[8]}$ analyzed the torque transmission capability of magneto-rheological fluid clutch and found out that the torque increases with the increase of magnetic field strength; Zhengjun et al. ${ }^{[9,10]}$ analyzed the flow model of MRF in the driving device and the fluid dynamic characteristics of magneto-rheological transmission device.

\subsection{Magnetorheological Fluid Shock Absorber}

Dyke. S.J、D.F.Spence. Jr、J.D. Carlson and M.K. Sain et al. ${ }^{[11,12]}$ from America made full use of various excellent properties of MRF, studied on the application of MRF damper in structural vibration control deeply, thus completed the design model of MRF shock absorber which can be applied to seismic protection of buildings and bridges. The results showed that the MRF shock absorber can satisfy the requirement of building seismic protection, and meet all kinds of constraints related.
Moreover, Farban Gamdhi et al.from Pennsylvania State University used MRF shock absorber for vibration control of helicopter rotor blade, and the experimental results showed that it can greatly reduce the blade tremor to improve its stability. Carlson and his partner Dyke. S.J studied on a controllable MRF shock absorber for truck seat ${ }^{[13]}$, and let it directly instead of common shock absorber to make the amplitude $20 \% \sim 50 \%$ lower, reduce the risk of traffic along the mountain road greatly .

\section{Magnetorheological Fluid Application Prospects}

Engine dynamometer offers load for the output power of the test machine, takes in mechanical energy output by the test machine, Simultaneously the dynamic performance parameters of the test machine such as torque and rotational speed and so on is measured. Dynamometer is based on the principle that action and reaction are equal and opposite direction to measure torque. According to the characteristics of MRF, making full use of its fast response characteristics, a MRF engine dynamometer device is put forward. Fig.1 is the test bench of MRF engine dynamometer.

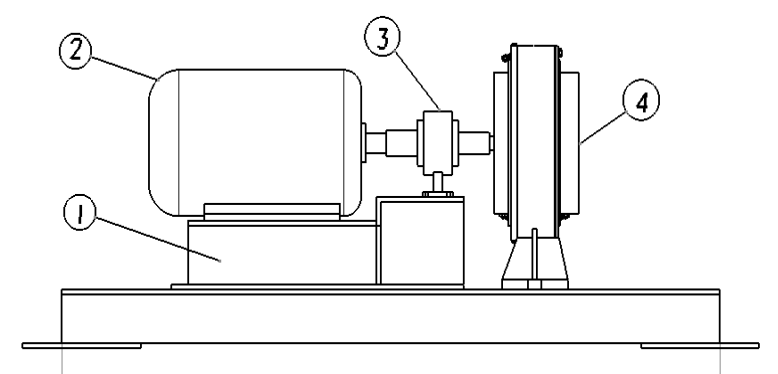

(1)Frame; (2)Rotating parts; (3)Torque sensor; (4)MRF engine dynamometer device

Fig 1. The test bench of MRF engine dynamometer.

MRF engine dynamometer relies mainly on the shear stress of MRF to transmit torque, it mainly includes excitation coil, shaft, turntable, shells etc, MRF exists between turntable and shell. With the action of magnetic field, Magnetorheological effect of MRF occurs, which makes its viscosity increase rapidly, and drag torque is applied in the objects that moves in the MRF, drag torque is associated with the magnetic induction intensity and the structure size of the device, and the magnetic induction intensity is associated with the current of excitation coil. Therefore, as long as the current value of excitation coil and the structure size of the device have been known, the size of the resistance moment can be got, so the output torque of rotating parts can be got. Rotating speed is obtained by torque sensor.

By adjusting the value of load current, the magnetic induction intensity of excitation coil can be changed, in order to achieve a adjustable magneto rheological fluid 
shear stress. With the increase of current value, magnetic induction intensity increases and magneto-rheological fluid shear stress increases, so leading to the load and the drag torque increase. Within a certain amount of current value, the dynamic performance parameters of the test machine such as torque and power can be got by measuring and calculating. According to the related theories and features of existing magneto-rheological fluid products, this dynamometer is a disk type magneto-rheological fluid dynamometer.

\section{Conclusion}

Because of its good mechanical properties, a wide temperature control range and magneto rheological effect etc, MRF has got more and more attentions and applications in the domain of engineering, especially in automobile field. According to the characteristics of magneto rheological fluid, a MRF engine dynamometer is presented, which hasn't been seen in the current research results.Although a more detailed and specific instruction about this MRF engine dynamometer is not given, the further research of it will continue and more information will be put forward in the near future.There is no doubt that the putting forward of this MRF engine dynamometer can inject fresh blood for magneto-rheological fluid devices available, widen road for the development of engine dynamometer, and supply more opportunities for further extensive use of magneto-rheological fluid.

\section{Acknowledgements}

This research was supported by the construction of professional platform for modern automobile service engineering in Shanghai city (XKCZ1214).

\section{References}

[1] Zuo Jun, Luo Xiaoyu, Cao Shupin. The magneto rheological fluid_—A new intelligent fluid mat- erial[J].Machine tool \& hydraulics, 2000(1): 30 .
[2] Yang Shi Qing, Zhang Wanli. Study on the rheological properties of magneto-rheological fluid[J]. Functional materials, 1998, 29 (5): 550-552.

[3] J.Rabinow.The magnetic fluid clutch[J].AIEE Transaction, 1948, 67:1308-1315.

[4] Yang Shiqing, Peng Bin, Jiang Hongchuan. Study on Preparation and rheological properties of composite intelligent magneto-rheological fluid system[J].Materials engineering, 2000(9): 21-24.

[5] Carlson J D.Magnetorheological fluid actuators [A].In: Janocha H. Adaptronics and Smart Structures[C].Berlin: Springer-Verlag Berlin Heidelberg. 1999:180-195.

[6] Foister R T.MR fluid devices[P].USA:US Patent:5667715, 1997.

[7] Gopalswamy S, Linzell S M, Jones G L, et al. MR fluid clutch with minimized reluctance[P]. USA:US Patent:5896965, 1999.

[8] Yalcintas M.Magneto-rheological Fluid Based Torque Transmission Clutches[A], Proceedings of the International Offshore and Engineering Conference[C], Brest, 1999, (4):563-569.

[9] Zhen Jun, Zhang Zhaohui, Cao Xingjin. Flow analysis of magneto rheological fluid in the transmission device[J].Journal of Central South University, 2008, 39 (1): $1149-1154$.

[10] Zhen Jun, Zhang Zhaohui, Cao Xingjin. Numerical calculation of transient flow characteristics of magneto rheological fluid in the transmission device[J].Journal of Xi'an Jiao Tong University, 2007, 41 (9): 1053-1057.

[11] Dyke. S.J, Spencer. Jr. B. F, M.K, et al. Experimental Verificantion of Semi-Active Structural Control Strategies Using Acceleration Feedback[A]. Proc. the 3rd Int Conf. on Motion and Vibr. Control[C], Chiba, Japan, 1996, (3):291-296.

[12] Housner. G.W. Structural Control: Past, Present and Future[J].Journal of Engineering Mechanics, 1997, 123(9):897-971.

[13] Lord Corporation Rheonetic Linear Damper, Rd-1001/Rd-1004, Product Information Sheet, Lord core.Pub.1997. 